\title{
Monogenic hypercholesterolemia: new insights in pathogenesis and treatment
}

\author{
Daniel J. Rader, ${ }^{1}$ Jonathan Cohen, ${ }^{2,3}$ and Helen H. Hobbs ${ }^{3,4}$ \\ ${ }^{1}$ Department of Medicine and Center for Experimental Therapeutics, University of Pennsylvania School of Medicine, \\ Philadelphia, Pennsylvania, USA \\ ${ }^{2}$ Center for Human Nutrition, and \\ ${ }^{3}$ Eugene McDermott Center for Human Growth and Development and the Department of Molecular Genetics, \\ University of Texas Southwestern Medical Center, Dallas, Texas, USA \\ ${ }^{4}$ Howard Hughes Medical Institute, Dallas, Texas, USA
}

J. Clin. Invest. 111:1795-1803 (2003). doi:10.1172/JCI200318925.

\section{Introduction}

The careful clinical characterization of patients with genetic forms of severe hypercholesterolemia has played a critical role in the historic linkage of hypercholesterolemia to atherosclerosis. Elucidation of gene defects that cause severe hypercholesterolemia has provided molecular entrées into the biosynthetic and regulatory pathways that produce and eliminate cholesterol and has led to the development of potent pharmacological agents that dramatically reduce circulating levels of cholesterol. The last decade of the twentieth century culminated in the demonstration that pharmacological reductions in plasma cholesterol levels result in fewer cardiovascular events and reduce total mortality.

This review will summarize recent developments in our understanding of the molecular pathogenesis and treatment of monogenic forms of severe hypercholesterolemia, and some implications that these findings have for the management of common forms of hypercholesterolemia.

General overview of LDL metabolism. Cholesterol is a rigid, hydrophobic molecule that confers structural integrity to plasma membranes of vertebrate cells. Excess cellular cholesterol is esterified with fatty acids to form cholesteryl esters, which are either stored as lipid droplets in cells or packaged with other apolipoproteins to form VLDL in the liver and and chylo-

The Science in Medicine series is supported in part by a generous grant from the Doris Duke Charitable Foundation.

Address correspondence to: Helen H. Hobbs, Department of Molecular Genetics, University of Texas Southwestern Medical Center, 5323 Harry Hines Boulevard, Dallas, Texas 75390, USA. Phone: (214) 648-6724; Fax: (214) 648-7539;

E-mail: Helen.Hobbs@UTSouthwestern.edu.

Conflict of interest: The authors have declared that no conflict of interest exists.

Nonstandard abbreviations used: LDL receptor (LDLR); LDL-cholesterol (LDL-C); familial hypercholesterolemia (FH); familial defective apoB-100 (FDB); autosomal recessive hypercholesterolemia (ARH). microns in the intestine (Figure 1). The two major cholesterol-carrying lipoproteins in humans are LDL and HDL. Approximately $70 \%$ of circulating cholesterol is transported as LDL.

LDL is formed in the circulation from VLDL (Figure 1). The triglycerides and phospholipids of circulating VLDL are hydrolyzed by lipases anchored to vascular endothelial surfaces, forming cholesterolenriched VLDL remnant particles. Approximately half of the VLDL remnants are cleared from the circulation by LDL receptor-mediated (LDLR-mediated) endocytosis in the liver, and the remainder undergoes further processing to produce LDL. Most LDL is removed from the circulation after binding to the hepatic LDLR via apoB-100.

Plasma levels of LDL-cholesterol (LDL-C) are directly related to the incidence of coronary events and cardiovascular deaths. Approximately $50 \%$ of the interindividual variation in plasma levels of LDL-C is attributable to genetic variation (1). The major portion of this genetic variation is polygenic, reflecting the cumulative effects of multiple sequence variants in any given individual. A subset of patients with very high plasma LDL-C levels have monogenic forms of hypercholesterolemia, which are associated with the deposition of cholesterol in tissues, producing xanthomas and coronary atherosclerosis.

The clinical features, diagnosis, and pathophysiology of the known mendelian disorders of severe hypercholesterolemia will be serially reviewed (Table 1). This will be followed by a discussion of how insights gleaned from the study of these disorders may be extended to the treatment of hypercholesterolemia in the general population.

\section{Familial hypercholesterolemia}

Historical perspective. Familial hypercholesterolemia $(\mathrm{FH})$, the most common and most severe form of monogenic hypercholesterolemia, was the first genetic disease of lipid metabolism to be clinically and 


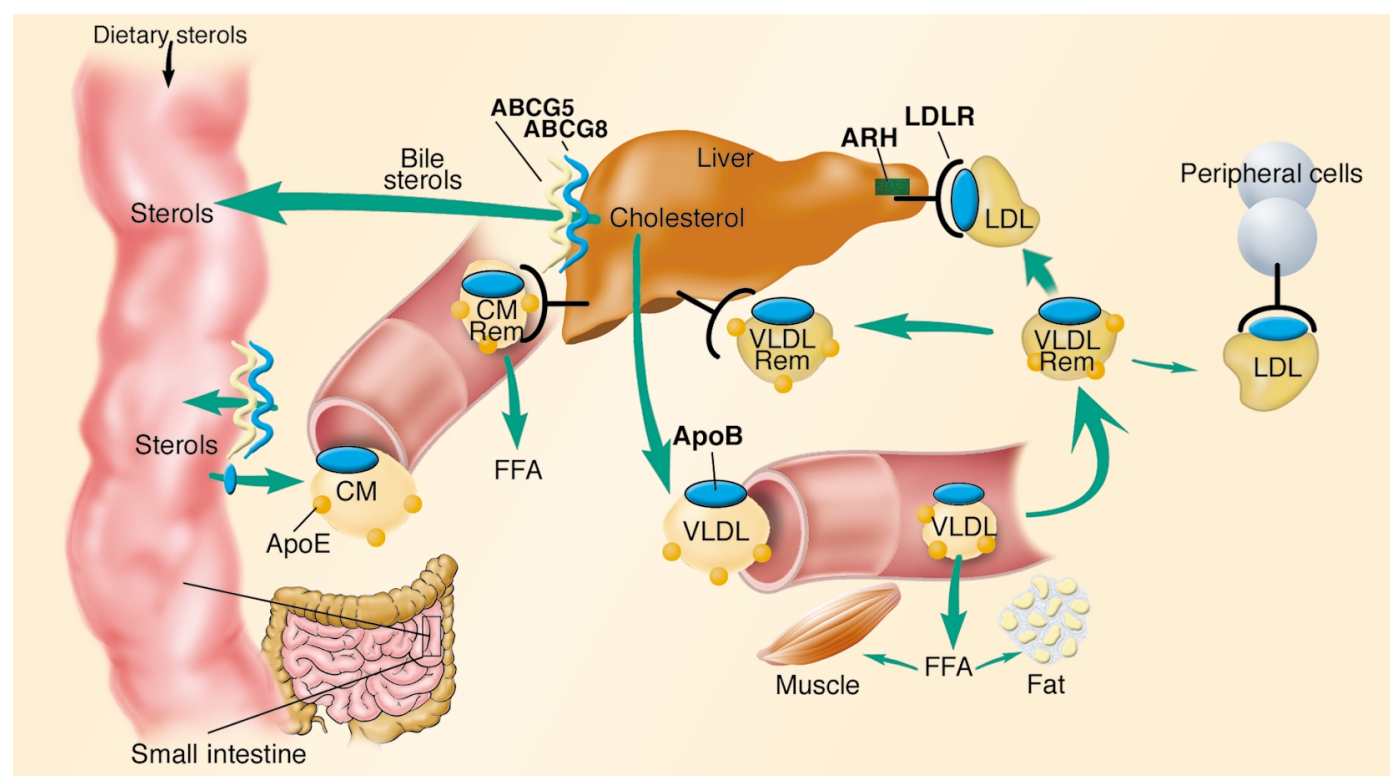

\begin{abstract}
Figure 1
Overview of LDL metabolism in humans. Dietary cholesterol and triglycerides are packaged with apolipoproteins in the enterocytes of the small intestine, secreted into the lymphatic system as chylomicrons (CM). As chylomicrons circulate, the core triglycerides are hydrolyzed by lipoprotein lipase, resulting in the formation of chylomicron remnants (CM Rem), which are rapidly removed by the liver. Dietary cholesterol has four possible fates once it reaches the liver: it can be esterified and stored as cholesteryl esters in hepatocytes; packaged into VLDL particles and secreted into the plasma; secreted directly into the bile; or converted into bile acids and secreted into the bile. VLDL particles secreted into the plasma undergo lipolysis to form VLDL remnants (VLDL Rem). Approximately $50 \%$ of VLDL remnants are removed by the liver via the LDLR, and the remainder mature into LDL, the major cholesterol transport particle in the blood. An estimated $70 \%$ of circulating LDL is cleared by LDLR in the liver. ABCG5 and ABCG8 are located predominantly in the enterocytes of the duodenum and jejunum, the sites of uptake of dietary sterols, and in hepatocytes, where they participate in sterol trafficking into bile. Mutations in either transporter cause an increase in delivery of dietary sterols to the liver and a decrease in secretion of sterols into the bile. Autosomal recessive hypercholesterolemia $(\mathrm{ARH})$ is a putative adaptor protein that is involved in the mechanics of LDLR-mediated endocytosis. ABCG: ATP-binding cassette, family G.
\end{abstract}

molecularly characterized (2). The disease has an autosomal codominant pattern of inheritance and is caused by mutations in the $L D L R$ gene; individuals with two mutated LDLR alleles (FH homozygotes) are much more severely affected than those with one mutant allele (FH heterozygotes). The plasma levels of LDL-C are uniformly very high in FH homozygotes, irrespective of diet, medications, or lifestyle. For example, FH homozygotes living in China, where the dietary intake of cholesterol and saturated fat is low, have plasma LDL-C levels similar to those of FH homozygotes living in Western countries (3).

FH homozygotes develop cutaneous (planar) xanthomas and coronary atherosclerosis in childhood (2). Atherosclerosis develops initially in the aortic root, causing supravalvular aortic stenosis, and then extends into the coronary ostia. The severity of atherosclerosis is proportional to the extent and duration of elevated plasma LDL-C levels (calculated as the cholesterol-year score) (4). If the LDL-C level is not effectively reduced, $\mathrm{FH}$ homozygotes die prematurely of atherosclerotic cardiovascular disease. Optimization of other cardiovascular risk factors has little impact on the clinical course of the disease.

Patients with homozygous $\mathrm{FH}$ are classified into one of two major groups based on the amount of
LDLR activity measured in their skin fibroblasts: patients with less than $2 \%$ of normal LDLR activity (receptor-negative), and patients with $2-25 \%$ of normal LDLR activity (receptor-defective) (2). In general, plasma levels of LDL-C are inversely related to the level of residual LDLR activity. Untreated, receptornegative patients with homozygous $\mathrm{FH}$ rarely survive beyond the second decade; receptor-defective patients have a better prognosis but, with few exceptions, develop clinically significant atherosclerotic vascular disease by age 30 , and often sooner (2).

The plasma levels of LDL-C in FH heterozygotes are lower (elevated two- to threefold) and much more dependent on other genetic and environmental factors than are those in FH homozygotes. Although the nature of the molecular defect has some impact on the severity of hypercholesterolemia, FH heterozygotes with the same LDLR mutation can have widely different plasma levels of LDL-C (2). The clinical prognosis of FH heterozygotes is related not only to the magnitude of the elevation in plasma LDL-C but also to the presence of other coronary risk factors (5).

New insights into pathogenesis. Despite our detailed knowledge of the molecular biology of the LDLR, fundamental questions regarding how the receptor delivers its cargo in cells without being degraded itself have 
Table 1

Major monogenic diseases that cause severe hypercholesterolemia

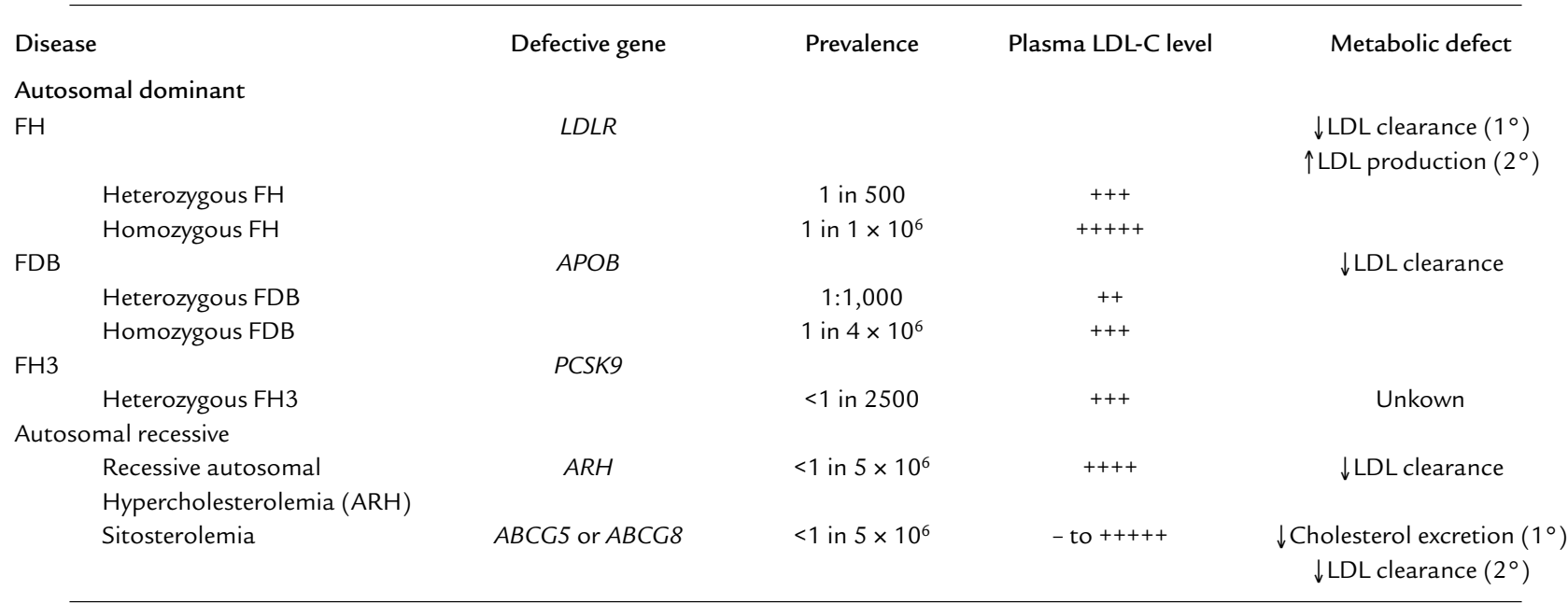

The number of + signs indicates the relative plasma level of LDL-C. $1^{\circ}$, primary major mechanism; $2^{\circ}$, secondary mechanism. FH, familial hypercholesterolemia; FDB, familial defective apoB-100; LDLR, LDL receptor.

only recently been elucidated. The crystal structure of the extracellular domain of the protein has provided a compelling model of how the receptor binds LDL with high affinity at the cell membrane and then releases it in the appropriate intracellular compartment (6). The extracellular domain consists of a ligand-binding domain, an EGF precursor homology domain, which contains a six-bladed $\beta$-propeller flanked by cysteine-rich EGF repeats (7), and an O-linked sugar-rich domain. When the LDLR is on the cell surface, the extracellular domain is extended, exposing the ligand-binding domain to LDL. After the receptor binds LDL, the receptor-ligand complex is internalized and delivered to endosomes. In the acidic environment of the endosome, the LDLR folds back on itself, bringing the $\beta$-propeller region of the EGF precursor domain into close apposition to the ligandbinding domain, thus displacing LDL. The $\beta$-propeller appears to function as a pseudosubstrate for the ligand-binding domain, permitting release of the lipoprotein in the endosome and recycling of the LDLR to the cell surface. Naturally occurring LDLR mutations in humans that disrupt recycling of the receptor are located in residues critical to the structure of the EGF precursor domain $(6,7)$.

LDL turnover studies documented the key role of hepatic LDLRs in LDL catabolism (2). More recent studies indicate that the LDLR may also regulate the rate of entrance of VLDL into the circulation. Mice overexpressing the lipogenic transcription factor SREBP-1a have increased hepatic cholesterol and triglyceride synthesis, and hepatic steatosis, but normal plasma lipid levels. When LDLR expression is abolished in these mice, they become profoundly hyperlipidemic due to an increase in secretion of apoB-containing lipoproteins (8). Evidence from studies in cultured mouse hepatocytes suggests that the LDLR may also restrict hepatic apoB-100 secretion by promoting its intracellular degradation (9). Thus, the LDLR may limit the number of circulating triglyceride-rich particles by reducing their secretion and by promoting their recapture before they enter the circulation from the liver. The importance of the LDLR in limiting VLDL secretion in vivo in humans remains controversial, because isotopic studies cannot readily determine the proportion of newly formed VLDL that enters the systemic circulation.

Diagnosis. In general, the diagnosis of FH is straightforward and is based on a family history of hypercholesterolemia and premature coronary atherosclerosis, the lipid profile, and the presence of xanthomas. Heterozygous FH occurs in approximately 1 in 500 persons worldwide but has a much higher incidence in certain populations, such as the Afrikaners, Christian Lebanese, Finns, and French-Canadians, due to founder effects (2). Over 900 mutations in the LDLR gene cause FH (10). Most mutations are unique, making the molecular diagnosis difficult, except in patients from populations where a limited number of mutations predominate. However, to date, there is no evidence that molecular diagnosis of the disease has important therapeutic implications.

Treatment. Heterozygous FH patients are responsive to statins, which inhibit HMG-CoA reductase and result in upregulation of the normal LDLR allele. Combination therapy is frequently required to achieve desired LDL-C levels (Table 2). Historically, bile acid sequestrants or nicotinic acid have been used for this purpose. Stanol esters, which decrease cholesterol absorption by displacing cholesterol from mixed micelles, are also effective in combination therapy (11). Recently, ezetimibe, a drug that specifically inhibits cholesterol absorption, became available (12). Ezetimibe binds to the microvilli of jejunal 
Table 2

Major LDL-C-lowering therapies for severe hypercholesterolemia

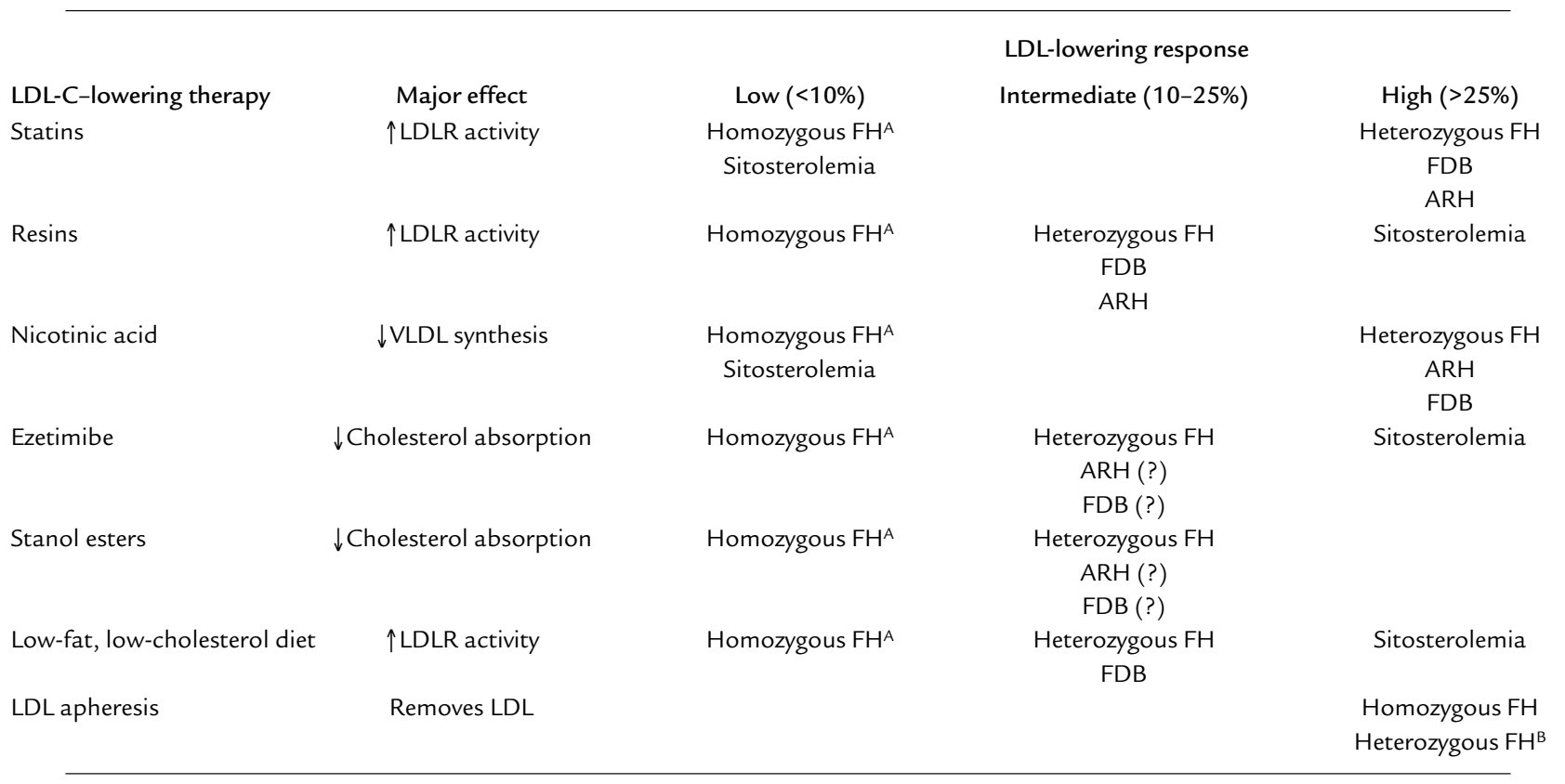

AFH homozygotes with no LDLR function. FH homozygotes who have residual LDLR function can have intermediate responses to the lipid-lowering agents listed. BPlasmapheresis is used in $\mathrm{FH}$ heterozygotes who do not reach the target LDL-C on maximum tolerated doses of lipid-lowering agents. ?, no data available.

enterocytes and interferes with the enterohepatic circulation of cholesterol, much as bile acid sequestrants interrupt the enterohepatic circulation of bile acids. The drug is effective at very low concentrations and so presumably works by inhibiting a putative cholesterol transporter. Ezetimibe is more effective than dietary cholesterol restriction in lowering plasma cholesterol levels because it decreases the uptake of both dietary and biliary cholesterol. The reduced flux of cholesterol from the gut to the liver leads to a compensatory increase in hepatic LDLR, resulting in an approximately $20 \%$ reduction in LDL-C. The discovery of ezetimibe has rejuvenated the study of intestinal cholesterol absorption.

HMG-CoA reductase inhibitors and ezetimibe have only modest effects on plasma levels of LDL-C in FH homozygotes, even when administered at high doses (13). While some FH homozygotes with receptordefective mutations may retain sufficient LDLR activity to respond to these potent lipid-lowering agents, drug therapy alone is never adequate treatment for these patients. The current treatment of choice for homozygous FH (and for heterozygotes whose plasma LDL-C remains elevated with drug therapy) is LDL apheresis. This process, in which the LDL particles are selectively removed from the circulation through extracorporeal binding to either dextran sulphate or heparin, can promote regression of xanthomas and may slow the progression of atherosclerosis (14). However, the procedure is time consuming and expensive and must be performed every 1-2 weeks. Although it retards the development of atherosclerosis, it does not prevent it, because of the recurrent hypercholesterolemia between procedures. Therefore, new therapies are urgently needed to treat the hypercholesterolemia of individuals suffering from homozygous $\mathrm{FH}$. Inhibition of microsomal transfer protein (MTP), which is required for synthesis of apoB-containing lipoproteins, reduces plasma cholesterol levels in LDLR-deficient rabbits (15) and may be effective in homozygous $\mathrm{FH}$, though the development of hepatic steatosis may be dose limiting. Liver-directed gene transfer of the LDLR theoretically is attractive but awaits the development of better and safer vectors.

\section{Familial defective apoB-100}

Historical perspective. A subset of individuals with a clinical presentation similar to $\mathrm{FH}$ and reduced rates of LDL catabolism were found to have normal LDLR activity. When LDL from these patients was infused into normal subjects, the heterologous LDL was cleared at a reduced rate compared with normal autologous LDL (16). The disease, familial defective apoB-100 (FDB), results from a missense mutation (Arg3500Gln) in the LDLR-binding domain of apoB-100 (17). Other less frequent mutations in $A P O B$ can also cause this disease. FDB occurs with a frequency of about 1 in 1,000 in Central Europe but is much less common in other populations (18). Like FH, FDB is characterized by elevated plasma LDL-C levels with normal triglycerides, tendon xanthomas, and premature atherosclerosis. The mean concentration of LDL-C is about $100 \mathrm{mg} / \mathrm{dl}$ higher in patients with FDB than in age 
matched controls, but the levels vary over a wide range. Over $25 \%$ of Europeans with FDB have plasma LDL-C levels below the 95th percentile of the population, which is a significantly higher percentage than is seen for $\mathrm{FH}$ (19).

FDB homozygotes have levels of plasma LDL-C comparable to those in $\mathrm{FH}$ heterozygotes rather than those in FH homozygotes (18). The increased risk of atherosclerosis in FDB homozygotes confirms that LDL itself is atherogenic, since there is no accumulation of remnant particles in these patients. The development of coronary disease is slower, however, in FDB homozygotes than in $\mathrm{FH}$ homozygotes, since most FDB homozygotes identified to date are between 40 and 60 years of age (18).

New insights into pathogenesis. LDL from FDB homozygotes binds the LDLR with approximately $10 \%$ of normal affinity and is removed from the circulation at one-third to one-quarter of the normal rate (20). As a consequence of the delayed clearance of the defective LDL, the circulating LDL in FDB heterozygotes contains significantly more particles bearing the mutant apoB-100 than particles bearing the normal apoB-100 (20). The results of kinetic studies using stable isotopes are consistent with the idea that the lower plasma LDL concentrations in FDB than in FH result from reduced rates of LDL production (21). Patients with FDB are able to clear LDL precursor particles, since remnant uptake is mediated by apoE rather than apoB-100 (Figure 1).

Diagnosis. FDB cannot be clinically distinguished from heterozygous $\mathrm{FH}$, although patients with $\mathrm{FDB}$ tend to have lower levels of plasma LDL-C and fewer xanthomas $(18,19)$. The single base pair substitution in the apoB-100 gene responsible for the most common missense mutation can be easily detected using a variety of molecular techniques (18), although absence of the sequence variation does not rule out the diagnosis of FDB, since some patients have other mutations in $A P O B$. The clinical management of FDB and heterozygous $\mathrm{FH}$ is similar; thus, it is not necessary to establish the molecular diagnosis of FDB.

Treatment. It had been anticipated that patients with FDB might be less responsive to statin therapy than patients with FH, but this is not the case. FDB heterozygotes and homozygotes are responsive to the LDL-lowering effects of statins, presumably because of increased clearance of VLDL remnants (Table 2).

\section{Other autosomal dominant forms of hypercholesterolemia}

Recently the molecular defect responsible for an additional form of autosomal dominant hypercholesteremic has been identified. These patients are clinically indistinguishable from patients with heterozygous FH and FDB (except in one family in which the hypercholesterolemic family members had a lower body mass index [ref. 22]; however, the disease does not segregate with either $L D L R$ or $A P O B$
$(22,23)$. The disease causing gene PCSK 9 encodes neural apoptosis-regulated convertase 1 (NARC-1), a member of the proteinase K family of subtilases (24, $25)$. Very little is known about this protein and elucidation of its function should provide interesting new insights into the control of plasma LDL-C.

\section{Autosomal recessive forms of hypercholesterolemia} Historical perspective. In 1973, Khachadurian described an unusual Lebanese family in which all four offspring had clinical features of homozygous FH, including severe hypercholesterolemia and large tendon xanthomas, and yet LDLR function in their cultured fibroblasts was near normal (26). Subsequent characterization of Sardinian patients with a similar phenotype revealed that LDL clearance rates were as low as in patients with homozygous $\mathrm{FH}$, and yet LDLR function in cultured fibroblasts was largely preserved (27). The hypercholesterolemia failed to segregate with either the $L D L R$ or the $A P O B$ gene; the new disorder was named autosomal recessive hypercholesterolemia (ARH).

New insights into pathogenesis. ARH is caused by mutations in the $A R H$ gene, which encodes a novel adaptor protein (28). To date, ten ARH mutations have been described $(29,30)$. All ten mutations interrupt the reading frame, precluding synthesis of a full-length protein. Although LDLR function is preserved in ARH fibroblasts, it is defective in transformed lymphocytes from these patients (31). The amount of LDLR is normal, but the distribution of immunodetectable receptor protein is significantly altered in ARH lymphocytes, with most of the LDLR residing on the plasma membrane (31). Cell surface LDL binding is increased, but LDL degradation is markedly reduced, indicating that $\mathrm{ARH}$ is involved in the internalization of the LDLR-LDL complex.

ARH contains an approximately 130 -residue phosphotyrosine-binding domain, which is present in several adaptor proteins that bind a sequence motif (NPXY) in the cytoplasmic tails of a variety of cell surface receptors, including the LDLR (32). Brown, Goldstein, and colleagues showed that the integrity of the FDNPXY sequence is required for internalization of the LDLR (33). ARH binds to this motif in a sequence-specific manner (34). This sequence also binds inositol phospholipids, which may anchor the protein to the plasma membrane (35). The C-terminal portion of the protein contains a canonical clathrin box consensus sequence (LLDLE in the human sequence) that binds the heavy chain of clathrin, and a highly conserved 27-amino acid sequence that binds the $\beta_{2}$-adaptin subunit of AP- 2 , a structural component of clathrin-coated pits (34). Taken together, these data suggest that ARH functions as a modular adaptor protein linking the LDLR to the endocytic machinery of the coated pit.

The exact role of ARH in LDLR function is not known. ARH may be required to chaperone LDLRs to 
coated pits, or simply to anchor receptors in the pits during internalization. A naturally occurring mutation in the Y of the NPXY motif (Y807C) that prevents LDLR clustering in coated pits and, consequently, LDLR internalization, also prevents ARH binding to the cytoplasmic tail in vitro $(34,36)$. This finding suggests that ARH may play a role in LDLR clustering. Direct observations of LDLR trafficking in ARH-deficient cells will be required to determine whether this is indeed the case.

Why is ARH required for LDLR function in some cell types, such as hepatocytes, but not in others, such as fibroblasts? ARH is one of a large family of adapter proteins, others of which have been documented to associate with the LDLR tail (37). Perhaps one of these other family members can effectively substitute for ARH in fibroblasts, but not in hepatocytes. Alternatively, the process of LDLR internalization may differ in hepatocytes and fibroblasts. Unlike in fibroblasts, where LDLRs are clustered in coated pits, in hepatocytes LDLRs are dispersed on the plasma membrane (38). ARH may perform a specific function in hepatocytes that is not required in fibroblasts.

ARH appears to be a near perfect phenocopy of $\mathrm{FH}$ (39), which is consistent with the possibility that all clinical sequelae of ARH mutations result from defective LDLR activity. ARH may be involved in other receptor pathways, but no phenotypes indicating defective function of other NPXY-containing proteins have been reported in subjects with ARH.

Diagnosis. Plasma levels of LDL-C in ARH patients tend to be intermediate between those in FH heterozygotes and those in FH homozygotes (39). The onset of clinically significant coronary atherosclerotic disease is later in ARH patients than in patients with homozygous FH. Despite having lower plasma levels of cholesterol than FH homozygotes, patients with ARH often have large, bulky xanthomas $(26,39)$.

Treatment. Subjects with ARH respond to lipid-lowering medications with more substantial plasma cholesterol reductions than patients with homozygous $\mathrm{FH}$; in particular, statins produce striking reductions in plasma cholesterol in some ARH patients (39) (Table 2). The increased LDLR expression induced by statins may compensate to some degree for the defective hepatic LDLR function in ARH. Most ARH subjects do not reach optimal plasma cholesterol levels on lipid-lowering medications alone and are maintained on LDL apheresis (39).

\section{Sitosterolemia}

Historical perspective. In 1974, Bhattacharyya and Connor described two normocholesterolemic siblings with large xanthomas, elevated plasma levels of the major plant sterol sitosterol, and normolipidemic parents (40). In normal individuals, cholesterol constitutes more than $99 \%$ of circulating sterols; noncholesterol sterols, such as sitosterol, are present in only trace amounts. In sitosterolemia, plasma levels of sitosterol are elevated more than 50-fold, and approximately $15 \%$ of circulating and tissue sterols are derived from plants and shellfish (41).

Although the index cases of sitosterolemia were not hypercholesterolemic, most patients identified subsequently have had elevated plasma levels of LDL-C, especially in childhood. Children with sitosterolemia can have plasma LDL-C levels as high as those seen in FH homozygotes (>500 mg/dl) and also often develop planar xanthomas. Like patients with $\mathrm{FH}$, these patients can develop aortic stenosis and premature coronary atherosclerotic disease. A distinctive clinical feature of sitosterolemia is the occurrence of low-level hemolysis, presumably due to the incorporation of plant sterols into red blood membranes.

Sitosterol, though differing from cholesterol by only minor modifications of the side chain, has a very different metabolic fate (41). Both sitosterol and cholesterol are taken up into enterocytes in the proximal small intestine, and between $20 \%$ and $80 \%$ of dietary cholesterol is incorporated into chylomicrons. In contrast, less than $5 \%$ of dietary sitosterol is absorbed. The small amount of sitosterol that is transported to the liver is preferentially secreted into the bile (42). Consequently, plasma levels of plant sterols in normal individuals are very low. Patients with sitosterolemia have increased fractional absorption of dietary sterols and a defect in the ability to secrete sterols into the bile, resulting in the accumulation of both animal and plant sterols in the blood and body tissues (41). The plasma cholesterol level in patients with sitosterolemia is extremely responsive to dietary cholesterol restriction, which decreases the input of sterols into the body, and to bile acid resin therapy, which promotes the excretion of cholesterol by increasing bile acid synthesis.

Patients with sitosterolemia have strikingly low rates of cholesterol synthesis (41) and yet accumulate cholesterol in selected tissues, even when they are not hypercholesterolemic. The sterol composition of xanthomas and atherosclerotic lesions mirrors that of the plasma, so cholesterol is the predominant component. Although about $60 \%$ of the plant sterols in plasma are esterified, tissue noncholesterol sterols are present in the free form (40).

New insights into pathogenesis. Whereas the primary metabolic defect in FH, FDB, and ARH is in the receptor-mediated uptake of circulating LDL, sitosterolemia results from a defect in sterol efflux from cells. Sitosterolemia is caused by mutations in either of two adjacent genes that encode $\mathrm{ABC}$ half-transporters, ABCG5 and ABCG8 $(43,44)$. More than 25 different mutations cause sitosterolemia (43-45). Sitosterolemic patients invariably have two mutant alleles of $A B C G 5$ or two mutant alleles of $A B C G 8$ alleles; none of the patients identified has one mutation in $A B C G 5$ and one in $A B C G 8$. No obvious clinical differences in disease manifestation are apparent between patients with mutations in $A B C G 5$ or in 
$A B C G 8$. Interestingly, most Caucasian patients have mutations in $A B C G 8$, whereas all Japanese individuals with sitosterolemia identified to date have mutations in $A B C G 5$ (45).

$A B C G 5$ and $A B C G 8$ are expressed almost exclusively in hepatocytes and enterocytes and are coordinately upregulated by cholesterol feeding in mice (43), thus limiting the absorption and promoting the elimination of dietary sterols. The responses of $A B C G 5$ and $A B C G 8$ to cholesterol feeding are dependent on liver $\mathrm{X}$ receptor $\alpha(\operatorname{LXR} \alpha)$, a nuclear hormone receptor that orchestrates the regulation of several genes involved in the trafficking of cholesterol from tissues to the liver (ABCA1, APOE, CETP, and others) (46). The crucial role of ABCG5 and ABCG8 in cholesterol homeostasis has been revealed by genetic manipulation of the expression of the two genes in mice. Mice expressing no ABCG5 or ABCG8 provide an excellent animal model of sitosterolemia; the mice are sitosterolemic, have an increased fractional absorption of dietary sitosterol, and have markedly reduced levels of biliary cholesterol (47). Whereas the plasma cholesterol level increases only marginally in response to increases in dietary cholesterol in wild-type mice, plasma and liver cholesterol levels both increase dramatically in mice lacking ABCG5 and ABCG8.

Diagnosis. The diagnosis of sitosterolemia should be considered in individuals with xanthomatosis and hypercholesterolemia whose parents are normocholesterolemic. The disease should be suspected in hypercholesterolemic patients who have a greater than usual response to dietary cholesterol restriction or to bile acid resins (Table 2). The diagnosis of sitosterolemia is made by extraction of lipids from the plasma and fractionation of the sterols using gas-liquid chromatography.

Treatment. Sitosterolemic patients respond poorly to HMG-CoA reductase inhibitors but are unusually responsive to dietary cholesterol restriction and bile acid-binding resins (Table 2). Recently, ezetimibe, when combined with a low-sterol diet, was shown to be particularly effective at lowering plasma levels of plant sterols as well as cholesterol in sitosterolemic patients (48). This suggests that sitosterol and cholesterol enter the enterocyte by the same pathway. Whether ezetimibe will reduce the cardiovascular risk associated with sitosterolemia remains unknown. The optimal treatment for sitosterolemia is dietary sterol restriction, ezetimibe, and a bile acid sequestrant to limit the accumulation of both plant and animal sterols.

\section{Other recessive forms of hypercholesterolemia} Cholesterol $7 \alpha$-bydroxylase deficiency. Recently, Pullinger et al. described three hypercholesterolemic siblings (two brothers and a sister) with genetic deficiency of cholesterol $7 \alpha$-hydroxylase (CYP7A1), the first enzyme in the classical pathway for bile acid biosynthesis (49). The three affected siblings were homozygous for a frameshift mutation that results in pre- mature termination of translation and a nonfunctional enzyme. The two affected brothers were hypertriglyceridemic as well as hypercholesterolemic, but the levels of remnant particles in these patients were not determined. Both brothers were reportedly resistant to statin therapy, although plasma cholesterol levels normalized on a regimen of atorvastatin and niacin. The bile acid content of a 24-hour stool sample from a single proband was reduced by a striking $94 \%$, which is consistent with a dramatic reduction in bile acid synthesis.

$7 \alpha$-Hydroxylase deficiency presumably causes hypercholesterolemia by reducing hepatic LDLR activity. Evaluation of additional individuals with CYP7A1 deficiency will be necessary to confirm that the hypercholesterolemia results from the genetic defect in CYP7A1 and that the characteristic lipoprotein profile of this disorder is an isolated elevation in LDL-C. However, the finding that three siblings homozygous for the same mutation were all hypercholesterolemic suggests that disposal of cholesterol via the bile acid biosynthetic pathway is essential for the maintenance of normal plasma cholesterol levels in humans.

Thus, both major pathways for excretion of cholesterol into bile appear to be required for normal cholesterol homeostasis: cholesterol that accumulates because of defects in the ABCG5/ABCG8 transporter cannot simply be quantitatively excreted by conversion to bile acids, and direct secretion if cholesterol by ABCG5/ABCG8 cannot compensate for reduced bile acid synthesis.

\section{Implications for the treatment of hypercholesterolemia}

Insights derived from the study of patients with wellcharacterized monogenic disorders have implications for the management of hypercholesterolemia in the general population. The robust relationship between FH and premature coronary atherosclerotic disease provides the cornerstone supporting the primary role of elevations in plasma LDL-C levels in coronary atherosclerosis. Despite the inevitable development of clinically significant coronary atherosclerosis in $\mathrm{FH}$ homozygotes, the age of onset and severity of the disease vary widely, even among patients with identical LDLR mutations (50). These findings reflect the complexity of the atherosclerotic lesion and highlight the difficulties associated with predicting the clinical course of individual patients. Although the identification of DNA sequence variations associated with coronary atherosclerosis may provide windows into new therapeutic targets, it is highly unlikely that assaying single or even hundreds of DNA sequence polymorphisms will provide useful predictive information regarding disease risk (51). Changes in modifiable risk factors such as diet, cessation of smoking, weight and blood pressure control, and, of course, cholesterol reduction will have substantially more impact on the incidence and progression of atherosclerosis. 
Large-scale clinical trials provide convincing evidence that substantial LDL lowering reduces cardiovascular morbidity and mortality, even in subjects who do not have elevated LDL-C levels (52). Since an ever increasing proportion of the population would benefit from LDL-lowering therapy, can we develop ways to predict which agents will be most effective in a given individual? The statins, our most effective LDL-lowering agents, do not lower plasma cholesterol levels in patients with sitosterolemia, suggesting that these drugs may be less effective in patients who have increased cholesterol absorption and reduced hepatic cholesterol synthesis. Miettinen and colleagues have provided evidence that plasma levels of plant sterols may predict the relative responsiveness of individuals to two of the major classes of hypolipidemic agents: those that interfere with cholesterol synthesis (statins) and those that interfere with cholesterol absorption (ezetimibe and stanol esters) (53). Stanol esters are most effective in individuals with increased cholesterol absorption; conversely, statins may be less efficacious in these individuals, since the increased influx of dietary cholesterol to the liver is associated with lower rates of hepatic cholesterol biosynthesis (53). The interindividual differences in the response to various lipid-lowering agents illustrate the genetic heterogeneity underlying polygenic hypercholesterolemia. A better understanding of the molecular mechanisms causing these genetic differences may contribute to the development of new, more individualized therapies for the treatment of hypercholesterolemia.

\section{Acknowledgments}

D.J. Rader is a recipient of a Burroughs Wellcome Fund Clinical Scientist Award in Translational Research and a Doris Duke Distinguished Clinical Scientist Award. H.H. Hobbs is an Investigator of the Howard Hughes Medical Institute. This work was supported by grants from the NIH (HL-20948, HL72304, HL-53917, HL-55323, HL-59407, HL-70128, and RR-00040), the W.M. Keck Foundation, and the D.W. Reynolds Cardiovascular Clinical Research Center. We wish to thank Jay Horton and Scott Grundy for helpful discussions.

1. Heller, D.A., de Faire, U., Pedersen, N.L., Dahlen, G., and McClearn, G.E. 1993. Genetic and environmental influences on serum lipid levels in twins. N. Engl. J. Med. 328:1150-1156.

2. Goldstein, J., Hobbs, H., and Brown, M. 2001. Familial hypercholesterolemia. In The metabolic and molecular bases of inherited disease. C. Scriver, A. Beaudet, W. Sly, and D. Valle, editors. McGraw-Hill. New York, New York, USA. 2863-2913.

3. Sun, X.M., et al. 1994. Familial hypercholesterolemia in China. Identification of mutations in the LDL-receptor gene that result in a receptornegative phenotype. Arterioscler. Thromb. 14:85-94.

4. Hoeg, J.M., Feuerstein, I.M., and Tucker, E.E. 1994. Detection and quantitation of calcific atherosclerosis by ultrafast computed tomography in children and young adults with homozygous familial hypercholesterolemia. Arterioscler. Thromb. 14:1066-1074.

5. Hill, J.S., Hayden, M.R., Frohlich, J., and Pritchard, P.H. 1991. Genetic and environmental factors affecting the incidence of coronary artery disease in heterozygous familial hypercholesterolemia. Arterioscler. Thromb. 11:290-297.
6. Rudenko, G., et al. 2002. Structure of the LDL receptor extracellular domain at endosomal pH. Science. 298:2353-2358.

7. Jeon, H., et al. 2001. Implications for familial hypercholesterolemia from the structure of the LDL receptor YWTD-EGF domain pair. Nat. Struct. Biol. 8:499-504.

8. Horton, J.D., Shimano, H., Hamilton, R.L., Brown, M.S., and Goldstein, J.L. 1999. Disruption of LDL receptor gene in transgenic SREBP-1a mice unmasks hyperlipidemia resulting from production of lipid-rich VLDL. J. Clin. Invest. 103:1067-1076.

9. Gillian-Daniel, D.L., Bates, P.W., Tebon, A., and Attie, A.D. 2002. Endoplasmic reticulum localization of the low density lipoprotein receptor mediates presecretory degradation of apolipoprotein B. Proc. Natl. Acad. Sci. U. S. A. 99:4337-4342.

10. The low density lipoprotein receptor (LDLR) gene in familial hypercholesterolemia. www.Ucl.ac.uk/fh.

11. Miettinen, T.A., and Gylling, H. 1999. Regulation of cholesterol metabolism by dietary plant sterols. Curr. Opin. Lipidol. 10:9-14.

12. Van Heek, M., et al. 1997. In vivo metabolism-based discovery of a potent cholesterol absorption inhibitor, SCH58235, in the rat and rhesus monkey through the identification of the active metabolites of SCH48461. J. Pharmacol. Exp. Ther. 283:157-163.

13. Gagne, C., Gaudet, D., and Bruckert, E. 2002. Efficacy and safety of ezetimibe coadministered with atorvastatin or simvastatin in patients with homozygous familial hypercholesterolemia. Circulation. 105:2469-2475.

14. Thompson, G.R. 2003. LDL apheresis. Atherosclerosis. 167:1-13.

15. Wetterau, J.R., et al. 1998. An MTP inhibitor that normalizes atherogenic lipoprotein levels in WHHL rabbits. Science. 282:751-754

16. Vega, G.L., and Grundy, S.M. 1986. In vivo evidence for reduced binding of low density lipoproteins to receptors as a cause of primary moderate hypercholesterolemia. J. Clin. Invest. 78:1410-1414.

17. Soria, L.F., et al. 1989. Association between a specific apolipoprotein B mutation and familial defective apolipoprotein B-100. Proc. Natl. Acad. Sci. U. S. A. 86:587-591.

18. Myant, N.B. 1993. Familial defective apolipoprotein B-100: a review, including some comparisons with familial hypercholesterolaemia. Atherosclerosis. 104:1-18.

19. Hansen, P.S. 1998. Familial defective apolipoprotein B-100. Dan. Med. Bull. 45:370-382.

20. Innerarity, T.L., et al. 1990. Familial defective apolipoprotein B-100: a mutation of apolipoprotein B that causes hypercholesterolemia. J. Lipid Res. 31:1337-1349.

21. Schaefer, J.R., et al. 1997. Homozygous familial defective apolipoprotein B-100. Enhanced removal of apolipoprotein E-containing VLDLs and decreased production of LDLs. Arterioscler. Thromb. Vasc. Biol. 17:348-353.

22. Haddad, L., et al. 1999. Evidence for a third genetic locus causing familial hypercholesterolemia. A non-LDLR, non-APOB kindred. J. Lipid. Res. 40:1113-1122.

23. Varret, M., et al. 1999. A third major locus for autosomal dominant hypercholesterolemia maps to 1p34.1-p32. Am. J. Hum. Genet. 64:1378-1387.

24. Abifadel, M., et al. 2003. Mutations in PCSK9 cause autosomal dominant hypercholesterolemia. Nat. Genet. In press.

25 . Seidah, N.G., et al. 2003. The secretory proprotein convertase neural apoptosis-regulated convertase 1 (NARC-1): liver regeneration and neuronal differentiation. Proc. Natl. Acad. Sci. U. S. A. 100:928-933.

26. Khachadurian, A.K., and Uthman, S.M. 1973. Experiences with the homozygous cases of familial hypercholesterolemia. A report of 52 patients. Nutr. Metab. 15:132-140.

27. Zuliani, G., et al. 1995. Severe hypercholesterolaemia: unusual inheritance in an Italian pedigree. Eur. J. Clin. Invest. 25:322-331.

28. Garcia, C.K., et al. 2001. Autosomal recessive hypercholesterolemia caused by mutations in a putative LDL receptor adaptor protein. Science. 292:1394-1398

29. Cohen, J.C., Kimmel, M., Polanski, A., and Hobbs, H.H. 2003. Molecular mechanisms of autosomal recessive hypercholesterolemia. Curr. Opin. Lipidol. 14:121-127.

30. Eden, E.R., et al. 2002. Restoration of LDL receptor function in cells from patients with autosomal recessive hypercholesterolemia by retroviral expression of ARH1. J. Clin. Invest. 110:1695-1702. doi:10.1172/ JCI200216445

31. Norman, D., et al. 1999. Characterization of a novel cellular defect in patients with phenotypic homozygous familial hypercholesterolemia. J. Clin. Invest. 104:619-628.

32. Forman-Kay, J.D., and Pawson, T. 1999. Diversity in protein recognition by PTB domains. Curr. Opin. Struct. Biol. 9:690-695.

33. Davis, C.G., van Driel, I.R., Russell, D.W., Brown, M.S., and Goldstein, J.L. 1987. The low density lipoprotein receptor. Identification of amino acids in cytoplasmic domain required for rapid endocytosis. J. Biol. Chem. 262:4075-4082.

34. He, G., Gupta, S., Michaely, P., Hobbs, H.H., and Cohen, J.C. 2002. ARH is a modular adaptor protein that interacts with the LDL receptor, clathrin and AP-2. J. Biol. Chem. 277:44044-44049. 
35. Mishra, S.K., Watkins, S.C., and Traub, L.M. 2002. The autosomal recessive hypercholesterolemia (ARH) protein interfaces directly with the clathrin-coat machinery. Proc. Natl. Acad. Sci. U. S. A. 99:16099-16104.

36. Davis, C.G., et al. 1986. The J.D. mutation in familial hypercholesterolemia: amino acid substitution in cytoplasmic domain impedes internalization of LDL receptors. Cell. 45:15-24.

37. Gotthardt, M., et al. 2000. Interactions of the low density lipoprotein receptor gene family with cytosolic adaptor and scaffold proteins suggest diverse biological functions in cellular communication and signal transduction. J. Biol. Chem. 275:25616-25624.

38. Pathak, R.K., et al. 1990. Tissue-specific sorting of the human LDL receptor in polarized epithelia of transgenic mice. J. Cell Biol. 111:347-359.

39. Arca, M., et al. 2002. Autosomal recessive hypercholesterolaemia in Sardinia, Italy, and mutations in ARH: a clinical and molecular genetic analysis. Lancet. 359:841-847.

40. Bhattacharyya, A.K., and Connor, W.E. 1974. Beta-sitosterolemia and xanthomatosis. A newly described lipid storage disease in two sisters. J. Clin. Invest. 53:1033-1043.

41. Salen, G., et al. 1992. Sitosterolemia. J. Lipid Res. 33:945-955.

42. Salen, G., Ahrens, E.H., Jr., and Grundy, S.M. 1970. Metabolism of betasitosterol in man. J. Clin. Invest. 49:952-967.

43. Berge, K.E., et al. 2000. Accumulation of dietary cholesterol in sitosterolemia caused by mutations in adjacent ABC transporters. Science. 290:1771-1775.

44. Lee, M.H., et al. 2001. Identification of a gene, ABCG5, important in the regulation of dietary cholesterol absorption. Nat. Genet. 27:79-83.
45. Lu, K., et al. 2001. Two genes that map to the STSL locus cause sitosterolemia: genomic structure and spectrum of mutations involving sterolin-1 and sterolin-2, encoded by $A B C G 5$ and $A B C G 8$, respectively. Am. J. Hum. Genet. 69:278-290.

46. Repa, J.J., et al. 2002. Regulation of ATP-binding cassette sterol transporters ABCG5 and ABCG8 by the liver $\mathrm{X}$ receptors alpha and beta. J. Biol. Chem. 277:18793-18800.

47. Yu, L., et al. 2002. Disruption of Abcg5 and Abcg8 in mice reveals their crucial role in biliary cholesterol secretion. Proc. Natl. Acad. Sci. U. S. A. 99:16237-16242.

48. Salen, G., et al. 2002. Ezetimibe is an effective treatment for homozygous sitosterolemia. Circulation Supplement. 1l-185.

49. Pullinger, C.R., et al. 2002. Human cholesterol $7 \alpha$-hydroxylase (CYP7A1) deficiency has a hypercholesterolemic phenotype. J. Clin Invest. 110:109-117. doi:10.1172/JCI200215387.

50. Hobbs, H.H., Brown, M.S., Russell, D.W., Davignon, J., and Goldstein, J.L. 1987. Deletion in the gene for the low-density-lipoprotein receptor in a majority of French Canadians with familial hypercholesterolemia. N. Engl. J. Med. 317:734-737.

51. Hegele, R.A. 2002. Environmental modulation of atherosclerosis end points in familial hypercholesterolemia. Atheroscler. Suppl. 2:5-7.

52. 2002. MRC/BHF Heart Protection Study of cholesterol lowering with simvastatin in 20,536 high-risk individuals: a randomised placebo-controlled trial. Lancet. 360:7-22.

53. Gylling, H., and Miettinen, T.A. 2002. Baseline intestinal absorption and synthesis of cholesterol regulate its response to hypolipidaemic treatments in coronary patients. Atherosclerosis. 160:477-481. 